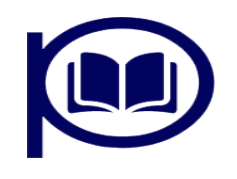

\title{
The impact of COVID-19 on children
}

\section{Aline Miranda Scovino ${ }^{1,2}$, Elizabeth Chen Dahab ${ }^{1}$ and Alexandre Morrot ${ }^{2,3 *}$}

\author{
${ }^{1}$ Department of Virology, Paulo de Goes Institute of Microbiology, Federal \\ University of Rio de Janeiro, Rio de Janeiro, Brazil. \\ 2Department of Immunology, Paulo de Goes Institute for Microbiology, Federal \\ University of Rio de Janeiro, Rio de Janeiro, Brazil. \\ ${ }^{3}$ Department of Immunology, Federal University of Rio de Janeiro, Rio de \\ Janeiro, Brazil.
}

\begin{abstract}
The COVID-19 severity is usually correlated to age, gender, obesity, and other comorbidities. Nevertheless, with the advance of the vaccination, the number of people infected with SARS-COV-2 is decreasing. On the other hand, children infection is becoming more recurrent, while having milder symptoms. One can argue that the child's innate immunity, trained by live vaccines and frequent infections, is more effective to control the infection. Besides, the COVID-19 development in children is associated with hyper inflammatory condition, the Multisystem Inflammatory Syndrome in Children (MIS-C). The purpose of this review is to overview the immunological aspects of COVID-19 in children and to discuss aspects related to vaccination protocols for children and their relevance to the control of the pandemic.

Keywords: COVID-19, SARS-CoV-2, MIS-C, Pathology.
\end{abstract}

\section{DESCRIPTION}

More than a year and a half after the onset of the pandemic, and with the various studies on COVID19 and SARS-CoV-2, we know that the worsening of the disease is associated with individual risk factors such as age, gender, cardiovascular disease, obesity, and other comorbidities ( $\mathrm{Wu}$ and McGoogan, 2020). For this reason, these risk groups were selected to be the first to be immunized, in an attempt to reduce the mortality rate from the disease.

With the development of different vaccines and the advance of vaccination campaigns, it was observed, especially in countries where vaccination is more advanced, such as Iraq and the United States, that there was a change in the profile of infected individuals. While at the beginning of the pandemic the greatest number of deaths and hospitalizations were elderly and persons with comorbidities, today we see a significant reduction in these cases and death in these groups (Mallapaty, 2020).
As younger age groups are being reached, we see a robust increase in the number of COVID-19 cases in youth and children aged $0-19$. For this reason, some countries are vaccinating children and adolescents. Why use this strategy to fight the pandemic, if in this age group the risks of developing severe forms of the disease remain low?

We know that children are more efficient at fighting SARS-CoV-2, so they are either asymptomatic or developing mild forms of the disease. A study carried out in China, with 44,762 positive tests for the virus, taken from laboratories, showed that of the total only $416(1 \%)$ and $549(1 \%)$ were from age-groups $<10$ and $10-19$, respectively (Wu and McGoogan, 2020).

Apparently, in general, children mount a very fast and effective innate immune response to the virus, preventing its replication and keeping the viral load very low, undetectable by the Polymerase Chain Reaction test (Tosif et al., 2020). But they are still able to develop antibodies against the virus, 
especially against the spike protein (Nogrady, 2020).

Why children are more effective at fighting SARSCoV-2 infection is not well defined. Probably because children have strong innate immune responses due to trained immunity (secondary to live-vaccines and frequent viral infections), leading to early control of infection, probably at the site of entry (Dhochak et al., 2020). However, this hypothesis is difficult to test, and for some other viral infections, as like influenza, it is not true. Another possibility is that the $\mathrm{T}$ lymphocytes of children and young people are naive and for this reason, they would more efficiently neutralize the infection. Meanwhile, a study comparing the response of children and adults to SARS-CoV-2 shows that memory $\mathrm{T}$ lymphocyte activity to the spike protein of the virus is more robust in adults than in children, so this may not be the response (Pierce et al., 2020).

There are also other possible explanations, such as the reduced number of ACE2 receptors in children's noses, which would make them receive a reduced amount of viral particles at the time of exposure to the virus. Or, because children are naturally reservoirs of other coronaviruses, which can induce the production of antibodies, which can cross-protect against SARS-CoV-24.

Although these data show that children are more resistant to infection, the literature also shows that many children develop a different form of COVID19, related to a Multisystem Inflammatory Syndrome in Children (MIS-C). The common features are: "middle-aged" children 6-9 with a persistent high spiking fever, abdominal pain, diarrhea, skin rash, and rapidly deteriorating clinical conditions with the onset of shock, without clear signs of dehydration. Other less common features were arthralgia, cough, meningism, conjunctivitis and reddened, and cracked lips (Ehrenfeld et al., 2020).

MIS-C is a hyper inflammatory condition associated with COVID-19. The pathological mechanisms of SARS-CoV-2 infection responsible for MIS-C are not known for sure. Some authors propose that it is not associated with the infection itself, but with later phenomena, such as the production of antibodies that induce Antibodydependent Enhancement (ADE), or the blocking of IFNs I and III by the virus can cause a late cytokine storm, which will be the cause of MIS-C (Rowley, 2020).

This syndrome is similar to Kawasaki's disease which is a systemic vasculitis that usually affects children under 5 years old and although selflimited, it can cause coronary artery aneurysm in a high percentage of cases (Burns JC, 2020).
Despite some very similar symptoms, MIS-C has some peculiar characteristics, the first being the age, the children with MIS-C are significantly older than children with KD. Other characteristics such as leukopenia, high levels of ferritin, C-reactive Protein (CRP) and ventricular natriuretic peptide (a marker of heart failure) as well as low platelet count are not characteristic of KD (Rowley, 2020).

Since the beginning of the pandemic, they have observed an unusually high number of critically ill patients with clinical features consistent with these diseases. The occurrence of such cases with a stereotyped clinical picture, which is rarely seen in children, during the peak of SARS-CoV2 infection in highly epidemic areas, points to a causal relationship (Ehrenfeld et al., 2020). Since the MIS$C$ can be severely manifested, the vaccination of children and adolescents is of paramount importance.

The vaccine from the pharmaceutical PfizerBioNTech is already being administered to children and young people between 12-17 years old. Data from clinical studies of this vaccine showed that the immune response of children aged 12-15 years, who received the two doses of the immunizing agent, developed higher levels of neutralizing antibodies against the virus (Tirar virgula) when compared to young people aged $16-26$ years. The PfizerBioNTech trial in adolescents recorded 18 cases in the placebo group, and none in those who got the vaccine. In March of this year, Pfizer-BioNTech and Moderna began clinical trials in children below this age, the aim is to know if younger children will respond in the same way, and what the recommended vaccine dose (Callaway E, 2020).

A meta-analysis study showed that children would be less susceptible to SARS-CoV-2, especially those under 12 years of age and once infected, young children including those between the ages of 0-5 are less likely to transmit the virus (Viner et al., 2020). A German study conducted after schools reopened after the lock down lockdown showed that infection was less common in children aged 6-10 years and higher in older children, adolescents, and adults working in schools. Furthermore, the potential for transmission increases as age increases (Im Kampe et al., 2020). Similar data were observed in similar studies in other countries, in the United States, the rate of infection is twice as high in children aged 1217 years as it is among 5-11 years old (Leeb et al., 2020).

Why children transmit less is still unknown, one possibility is that because they have smaller lungs they would release a smaller amount of aerosol, this occurs with tuberculosis, but the pathophysiological characteristics of the two diseases are different (Lewis, 2020). 
Despite these studies indicate that children transmit SARS-CoV-2 less efficiently than adults, the vaccination of the greatest number of potential transmitters is essential for the control of the pandemic, it would remove children as a potential reservoir for asymptomatic infections and would guard against the emergence of new variants (Callaway $E, 2020$ ). The vaccination of children is also essential, as despite being more resistant to infection, some may still develop the multi systemic syndrome, which in some cases is severe and even fatal (Ehrenfeld et al., 2020).

\section{REFERENCES}

Wu Z, McGoogan JM (2020). Characteristics of and important lessons from the coronavirus disease 2019 (COVID-19) outbreak in China: summary of a report of 72314 cases from the Chinese centre for disease control and prevention. JAMA. 323(13): 1239-1242.

Mallapaty S (2021). Will COVID become a disease of the young? Nature. 595(7867):343-344.

Tosif S, Neeland MR, Sutton P, Licciardi PV, Sarkar $S$, Selva KJ, Do LA, Donato C, Toh ZQ, Higgins $R$, Van de Sandt C (2020). Immune responses to SARS-CoV-2 in three children of parents with symptomatic COVID-19. Nat Commun. $11(1): 1-8$.

Nogrady B (2020). How kid's immune systems can evade COVID. Nature. 588(7838):382.

Dhochak N, Singhal T, Kabra SK, Lodha R (2020). Pathophysiology of COVID-19: why children fare better than adults? Indian J Pediatr. 87(7):537-546.

Pierce CA, Preston-Hurlburt P, Dai $Y$, Aschner CB, Cheshenko N, Galen B, Garforth SJ, Herrera NG, Jangra RK, Morano NC, Orner E (2020). Immune responses to SARS-CoV-2 infection in hospitalized paediatric and adult patients. Sci Transl Med. 12(564): eabd5487.
Ehrenfeld M, Tincani A, Andreoli L, Cattalini M, Greenbaum A, Kanduc D, Alijotas-Reig J, Zinserling $V$, Semenova $N$, Amital $H$, Shoenfeld $Y$ (2020). Covid-19 and autoimmunity. Autoimmun Rev. 19(8):102597.

Rowley AH (2020). Understanding SARS-CoV-2related multisystem inflammatory syndrome in children. Nat Rev Immunol. 20(8):453-454.

Burns JC (2002). Commentary: translation of Dr. Tomisaku Kawasaki's original report of fifty patients in 1967. Pediatr Infect Dis J. 21(11):993-995.

Lewis D (2021). Does vaccinating adults stop kids from spreading COVID too? Nature. 594(7868):482-483.

Callaway E (2021). COVID vaccines and kids: five questions as trials begin. Nature. 592(7856):670-671.

Viner RM, Mytton OT, Bonell C, Melendez-Torres GJ, Ward J, Hudson L, Waddington C, Thomas J, Russell S, Van Der Klis F, Koirala A (2021). Susceptibility to SARS-CoV-2 infection among children and adolescents compared with adults: a systematic review and meta-analysis. JAMA Pediatr. 175(2):143-156.

Im Kampe EO, Lehfeld AS, Buda S, Buchholz U, Haas W (2020). Surveillance of COVID-19 school outbreaks, Germany, March to August 2020. Euro Surveill. 25(38): 2001645.

Leeb RT, Price S, Sliwa S, Kimball A, Szucs L, Caruso E, Godfred-Cato S, Lozier M (2020). COVID-19 trends among school-aged childrenUnited States, March 1-September 19, 2020. MMWR Morb Mortal Wkly Rep. 69(39): 14101415. 